\title{
Design and Implementation of Multimedia Player on Android
}

\author{
Gang $\mathrm{Du}^{1, \text { a }}$, Qiang $\mathrm{Fu}^{1}$ \\ ${ }^{1}$ Beijing Information technology college, Beijing 100078,China \\ adugang@163.com
}

Keywords: Android, multimedia player, video system.

\begin{abstract}
The video player based on Android system to develop, and fit in Amlogic platform, has improved the user interface, on the basis of the basic playback functions, you can set resume play, audio track, play mode, subtitles, screen mode, video information, and other functions, and also has 3D playback capability.
\end{abstract}

\section{Introduction}

As a new operating system, Android system has been applied to different smart mode terminals by many Companies with Google's supports and its opening. But its features of multi-media are required to be enhanced to meet various demands [1].

The applications of audio and video technology are becoming more widely. Study of Audio-Video system is help for developing Media Player, Video Conferencing, Digital TV, Video on Demand, and so on. The research emphasis of Audio and Video systems is put on the Application Framework Layer on Android [2]. Types and efficiency of media formats supported by Audio-Video system can not meet people's needs. The kind of video player is relatively less on Android. So, research and expansion of Audio-Video system and player development are very essential.

With the rapid development of network technology and the replacement of equipment, especially the increasing popularity of $3 \mathrm{G}$ networks, more and more functions of multimedia applications are required [3]. As a relatively new operating system, the multimedia framework of Android is not perfect. As an open source system, version differentiation of Android is a serious problem. It is a research of great value to improve the integrity of the multimedia framework of Android platform, for it could reduce the workload of developers, and brings the users with better experience [4]. Therefore, the development of framework with strong decoding functions, extensive protocol support and high decoding efficiency, has become the inevitable trend of the Android multimedia framework. Android system frameworkis shown in Fig. 1.

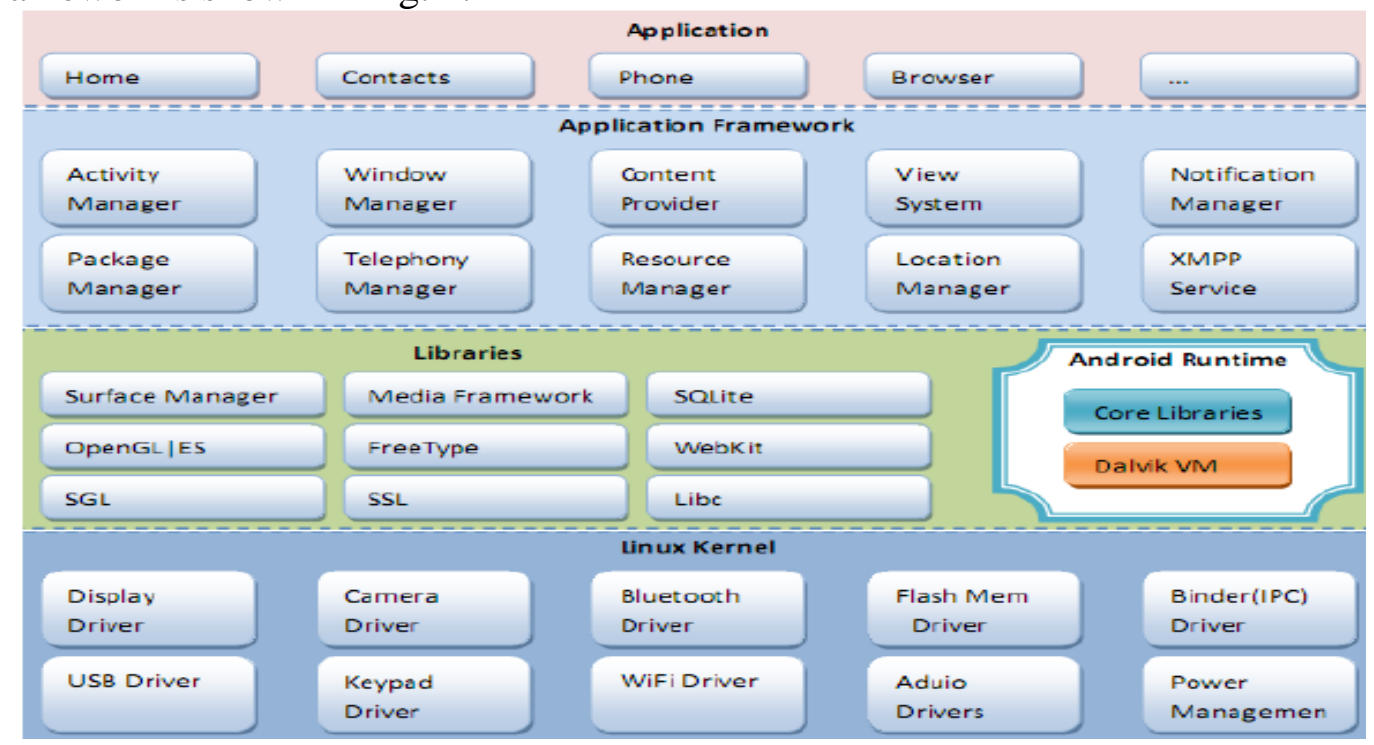

Fig. 1 Android system framework 


\section{Demand analysis}

With the development of hardware technology unceasing renewal, intelligent mobile phone such as bamboo shoots after a spring rain as into people's lives, we have notmeet the mobile phone can only be used for simple calls and send messages, requirements are more and more video, new development of technology have prompted further. The rapid development of mobile Internet era coming, 3G providesmany powerful condition for the development of video communication technology, the future of video communication in people's life, occupies an important position. This topic in this environment, the design can play streaming video player, video playbackon the phone to meet user requirements. In order to enhance Android multimedia system, two multimedia function extension methods are designed which from two aspect of sound and video. In order to achieve WMA decoding in the Android, this paper analysis of FF library, WMA file parsing and decoding part of the code in the FF is extracted and realizing transplantation to the Android system, so that the Android system supports the playback of WMA files. deeply analyzes and researches V 1 decoder chip interface and hardware decoding process of multimedia system of Android. Finally, based on the OpenMAX standard, Design a call to V1 decoder chip, and implement the hardware decode which based on the V 1 decoder chip. Through repeated play WMA audio files in the test machine of the Android platform, It has on reached the expected results that the play sound is clear and tone quality is good. Share of the smart phone market is shown in Fig. 2.

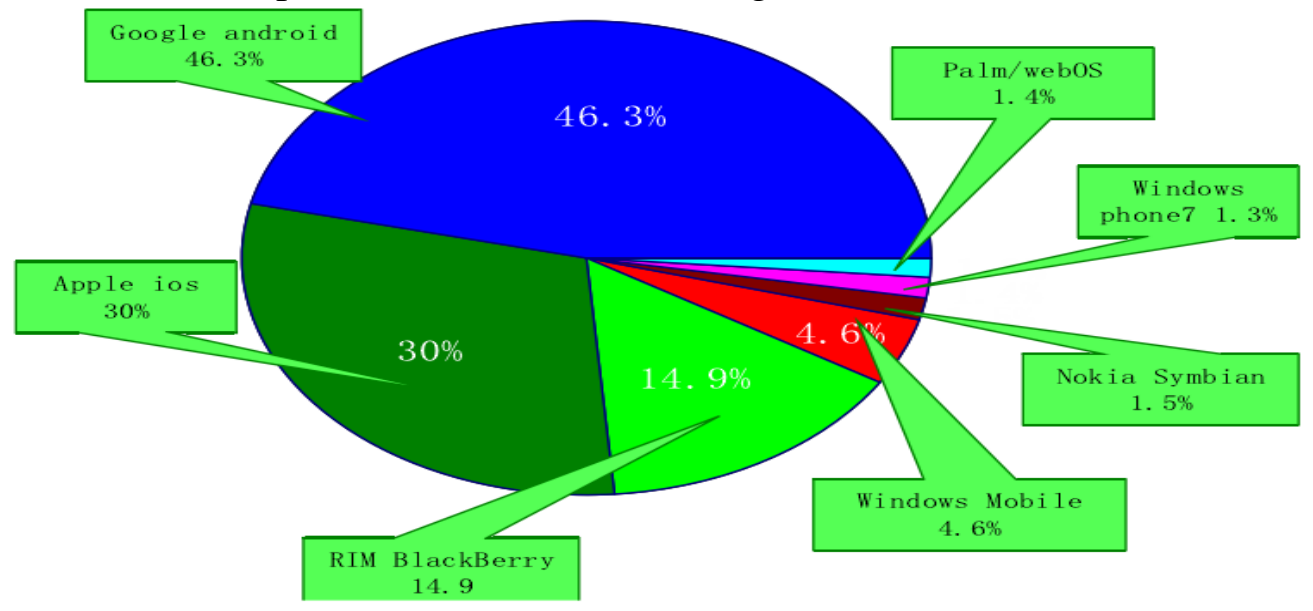

Fig. 2 Share of the smartphone market

\section{Multimedia design}

This paper topic is "mobile platform embedded software development, software developing embedded platform based on the Android mobile phone music player. Before the software design, first of all, a lot of work on android infrastructure information, and all kinds of music software on the market at present are analyzed, based on detailed analysis of the needs of users, and then according to the result of analysis was carried out for the whole software architecture and function module design, in terms of the UI done personalized design, improve user and player interaction, software function also added more humanized functions, e.g. electricity automatically suspended function, and using the freedom of the progress bar control music playback progress, etc. To implement the software based on Android framework, mainly implements the personalized Settings, playback controls, and other functional modules. Software implementation process, the author first use Eclipse to build the Android software development environment, using the Java language to write, code complete, has carried on the module to the functionality and performance of the software test, and the bugs found in the testing process was modified and optimized. Eventually determined by real machine test, the software has a small volume, strong portability, scalability is strong, and low requirements for terminal equipment configuration, suitable for a wide range of high school low-grade Android mobile phone users, but also can maintain the stability of long time running. Landroid.jar follows as Table 1. 
Table 1 Landroid.jar

\begin{tabular}{cc}
\hline Android.app & Android.os \\
\hline Android.content & Android.opengl \\
Android.database & Android.provider \\
Android.graphics & Android.telephone \\
Android.location & Android.view \\
Android.net & Android.util \\
Android.webkit & Android.widget \\
\hline
\end{tabular}

The hardware point of view, the general multimedia processing system includes codec unit processor and signal media stream. Some even joined the media information, separation of the components (Media Extractor), wherein the media stream processing that is the signal processing, after data reception to analyze its characteristics, and then accurately and rapidly for convolution, window, Fu Li transform large data computation, and outputs the result to the next component. Encoding and decoding unit is composed of a D/A and a A/D converter circuit two parts, concrete conversion operation to PCM (Pulse Code Modulation, pulse code modulation) form. First on the media continuous analog signal sampling, the discrimination (including two aspects of time and amplitude), the purpose is intuitively sample value with the most specific level close to the values indicated that there will be a continuous signal quantization. Finally the level value is represented by a binary or other form of code value, thus completing the coding process. Because of the realization of the process time and amplitude discrete more or less will cause the deviation of data or missing, resulting in media information defects and causes the distortion of the consequences. The decoding process is the process of player reverse execution and implementation: will save or transmission of the numerical code transformed by predicting or inverse techniques such as reduction, finally get the analog signal at the output, quality sound and picture quality after processing is directly determined by the quantization precision and algorithm. As shown in Fig, 3.

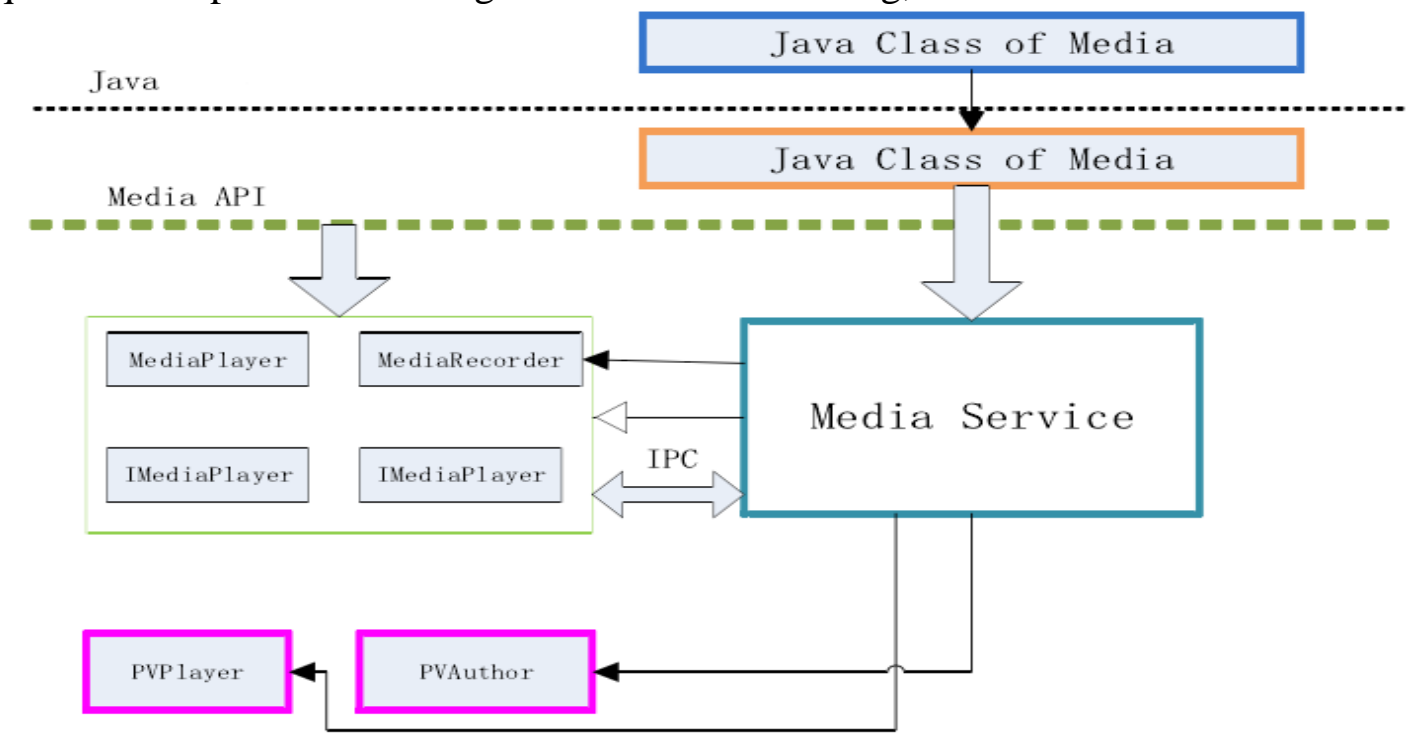

Fig. 3 Multimedia system structure

\section{Implementation and testing}

Experimental Jianzhong in eclipse ADT emulator Bu, we can according to the specificClick to select a different hardware conditions were simulated.

Codes are as follows:

int (*read_probe)(AVProbeData*);

int (*read_header)(structAVFormatContext*, AVFormatParameters *ap);

int (*read_packet)(structAVFormatContext*, AVPacket*pkt);

int (*read_close)(struct AVFormatContext*);

int (*read_seek)(struct AVFormatContext*, int stream_index, int64_t timestamp, int 
flags);

int (*write_header)(struct AVFormatContext*);

int $\left({ }^{*}\right.$ write_packet)(struct AVFormatContext*, AVPacket *pkt);

int (* write_trailer)(struct AVFormatContext*);

A new name for the ffmpeg Android project in eclipse, then the compiler generated libffmpeg.so, through NDK libjniaudio.so, copy the libjnivideo.so library file to the ffmpeg directory under the LIBS directory under the armeabi directory. Engineering at run time according to the related function automatically call.So library files in need,implement the operation. Video browser interface is shown in Fig. 4.

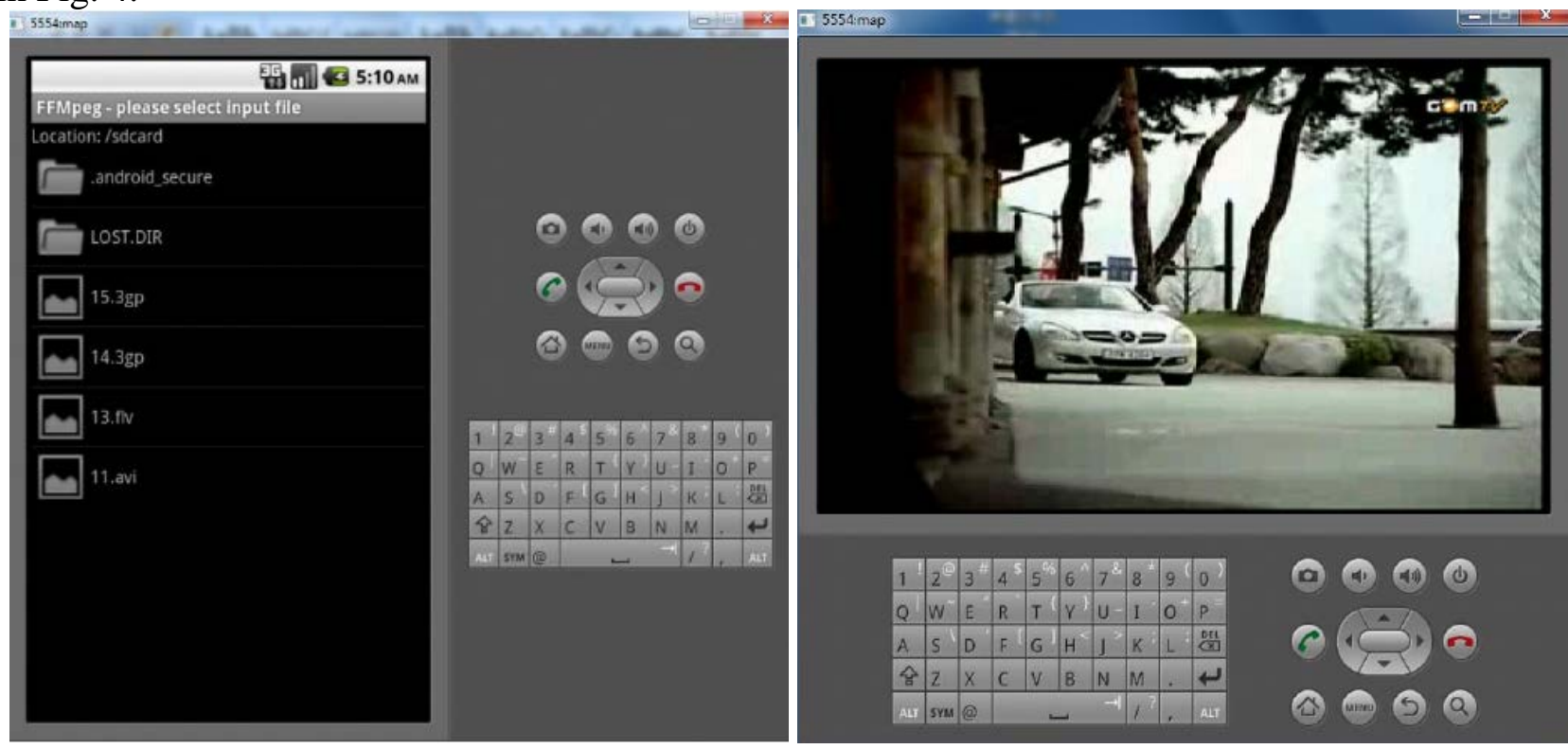

Fig. 4 Video browser interface

\section{Conclusions}

Sum up the experience in the development of the video player in Chinese and abroad, combined with the characteristics of the smart TV, smart set-top boxes and other equipment, proposed the design of the video player. First of all, study on the Android system, the Amlogic chip performance, hardware and software platform, Android application design, and then the progressive realization of the player interface package, background playback module design, and user interface design.

Final complete the main function of expected video player, achieved the video player which remote control and mouse as input, the TV screen as the output. The video player for high-definition player, smart TV, IP set-top boxes which output is TV screen. These video player fields are blank, and the video player also supports tablet and other touch devices, allows users in the most convenient way to control playback, and supports almost all formats and playback with rich functional.

\section{References}

[1] T Jarvenpaa, M Salmimaa. Optical characterization methods for autostereoscopic 3D displays. Journal of the Society for Information Display, 2008, 16(8): 825-833.

[2] Marko Gargenta. Learning Android. Sebastopol, California: o'Reilly Media, 2011: 6-53

[3] Lee Sangchul, Jeon JaeWook. Evaluating Performance of Android Platform Using Native C for Embedded Systems. Gyeonggido: 2010 International Conference on Control Automation and Systems International Conference, 2010: 1160-1163

[4] Information on http://www.khronos.org/openmax 